\title{
Metallic slit arrays filled with third-order nonlinear media: Optical Kerr effect and third-harmonic generation
}

\author{
Sergio G. Rodrigo, ${ }^{1}$ S. Carretero-Palacios, ${ }^{1}$ F. J. García-Vidal, ${ }^{2}$ and L. Martín-Moreno ${ }^{1}$ \\ ${ }^{1}$ Instituto de Ciencia de Materiales de Aragón and Departamento de Física de la Materia Condensada, \\ CSIC-Universidad de Zaragoza, E-50009 Zaragoza, Spain \\ ${ }^{2}$ Departamento de Física Teórica de la Materia Condensada, Universidad Autónoma de Madrid, E-28049 Madrid, Spain
}

(Received 15 February 2011; revised manuscript received 19 April 2011; published 29 June 2011)

\begin{abstract}
A theoretical study on the nonlinear optical properties of both gold and nickel slit arrays inlaid with a third-order nonlinear dielectric medium is presented. In particular, we investigate the optical Kerr effect and third harmonic generation. The geometrical parameters, along with the optical properties of each metal, determine the electromagnetic environment near the slits and, in turn, the nonlinear optical response. The optical Kerr effect is only seen for slit arrays in gold, where the transmitted and reflected currents are modified with the incident intensity, while nickel slit arrays behave linearly within the same range of input intensities. However, third harmonic emission occurs for both systems, unveiling third-order nonlinearities taking place also in nickel structures.
\end{abstract}

DOI: 10.1103/PhysRevB.83.235425

PACS number(s): 78.67.-n, 42.65.-k, 78.20.Bh

\section{INTRODUCTION}

Interaction of light with matter gives rise to a great number of nonlinear optical phenomena, such as Raman scattering, photoluminescence in metals, second harmonic (SH) generation, four wave mixing (FWM), third harmonic (TH) generation, or the optical Kerr effect (OKE). ${ }^{1}$ The nonlinear response of dielectrics has been long exploited in technological applications. In general, dielectrics in bulk present weak nonlinear response at moderately low laser powers. On the contrary, if metallic inclusions are present, the electric field can be efficiently enhanced and nonlinear effects in metallodielectric structures may occur at lower laser powers. ${ }^{2,3}$ Different configurations of metal slit arrays combined with nonlinear dielectrics have been widely investigated for their potential uses in optical bistability, ${ }^{4-6}$ nonlinear beam manipulation, ${ }^{7,8}$ and switching. ${ }^{9,10}$ Among the possible arrangements, an interesting example is a slit array filled with nonlinear dielectrics, investigated in Refs. 4,6, and 8 , where the nonlinear phenomena described originate from OKE, and in Ref. 11, where several aspects of harmonic generation have been analyzed. However, the physical mechanisms behind these two effects have not been correlated and to our knowledge, OKE and TH generation have not been investigated within the same theoretical framework (and for the same system) so far. Furthermore, the influence of absorption of metals in combination with nonlinear dielectrics and the relation to field enhancement have not been described either.

In this work, we investigate theoretically the optical response of metallic slit arrays inlaid with a Kerr-type nonlinear medium (see Fig. 1), where both TH generation and OKE phenomena are present. The former describes processes in which light at frequency $3 \omega$ is generated from an applied field at frequency $\omega$, whereas OKE processes have an influence on the fundamental harmonic, through a variation of the local dielectric constant. We also describe how the absorption of metals and slit widths affect the EM field near subwavelength slits which, in turn, determines the overall nonlinear response.
The paper is organized as follows: In Sec. II we describe the theoretical framework along with the numerical method used. In Sec. III we provide the geometrical and material parameters used for both the linear and nonlinear numerical simulations in Sec. IV and Sec. V, respectively. The later contains results for OKE (Sec. V A) as well as TH generation (Sec. V B). Finally, conclusions are presented in Sec. VI.

\section{THEORETICAL APPROACH}

We use the finite-difference time-domain (FDTD) method ${ }^{12}$ to treat both the linear optical properties of metals and the nonlinear third-order response of dielectrics. ${ }^{13}$ The third-order nonlinear dielectric is considered isotropic, homogeneous, and dispersionless in frequency, so the relationship between the displacement $(\vec{D})$ and the electric field $(\vec{E})$ is

$$
\begin{gathered}
\vec{D}=\varepsilon_{0} \vec{E}+\vec{P}, \\
\vec{P} \simeq \varepsilon_{0} \chi^{(1)} \vec{E}+\varepsilon_{0} \chi^{(3)}(\vec{E} \cdot \vec{E}) \vec{E}
\end{gathered}
$$

in the MKS system of units, where "." denotes the scalar product, $\varepsilon_{0}$ is the dielectric constant of vacuum, $\chi^{(1)}$ the linear susceptibility, and $\chi^{(3)}$ the third-order one.

In the particular case of harmonic fields, that is, $\vec{E}=$ $\vec{E}_{0}(\vec{r}) e^{-i \omega t}$, and taking into account that EM fields must be defined as real numbers, it can be easily found that ${ }^{1}$

$$
\begin{gathered}
\vec{P}_{R}=\varepsilon_{0}\left\{\chi^{(1)} \vec{E}_{R}+\frac{3 \chi^{(3)}}{4}|E|^{2} \vec{E}_{R}+\frac{\chi^{(3)}}{8}\left[\vec{E}^{3}+\left(\vec{E}^{*}\right)^{3}\right]\right\}, \\
\vec{D}_{R}=\varepsilon_{0}\left[\varepsilon+\frac{3 \chi^{(3)}}{4}|E|^{2}\right] \vec{E}_{R}+\frac{\varepsilon_{0} \chi^{(3)}}{8}\left[\vec{E}^{3}+\left(\vec{E}^{*}\right)^{3}\right],
\end{gathered}
$$

where $\vec{E}_{R}=\left(\frac{\vec{E}+\text { c.c. }}{2}\right)$ and $\varepsilon=1+\chi^{(1)}$, where c.c. stands for "complex conjugate." Note that the local change of the dielectric constant associated with OKE is given by the term $\Delta \varepsilon=\frac{3 \chi^{(3)}}{4}|E|^{2}$, whereas $\frac{\varepsilon_{0} \chi^{(3)}}{8}\left[\vec{E}^{3}+\left(\vec{E}^{*}\right)^{3}\right]$ describes the $\mathrm{TH}$ generation processes. 
In what follows we sketch how these expressions are implemented within a FDTD nonlinear algorithm. A full description of this algorithm can be found in Ref. 13. Let us start from the relation given by Eq. (1). Within the FDTD method, Maxwell's equations are discretized both in time and space. At each position inside the nonlinear medium, the displacement vector at instant $n+1, \vec{D}^{n+1}$, is obtained from its value at the previous instant $n$, and from the curl of the magnetic field at $n-1 / 2$. From Eq. (1), once $\vec{D}^{n+1}$ is known, the electric field at $n+1$ must satisfy

$$
\vec{E}^{n+1}=\frac{\vec{D}^{n+1}}{\varepsilon_{0}\left(\varepsilon+\chi^{(3)}\left|\vec{E}^{n+1}\right|^{2}\right)} .
$$

Taking the modulus of this expression, a cubic polynomial in $\left|\vec{E}^{n+1}\right|^{2}$ must be solved at each FDTD iteration. Because both $\varepsilon$ and $\chi^{(3)}$ are positive, there is only one real and positive root which must be chosen to ensure that $\left|\vec{E}^{n+1}\right|^{2}$ is real and positive.

In the FDTD method, illumination and data processing for retrieving the optical properties are key issues. Throughout this work we use $p$-polarized light sources at normal incidence [wave vector along the $z$ direction; see Fig. 1 (top)]. Unless otherwise stated, we employ in our calculations a gaussian wave packet $(\mathrm{GW})$, centered at a carrier frequency $\omega_{0}$. We are interested in nonlinear optical responses at a fixed wavelength, hence long-standing pulses (narrow in frequency) must be launched. Such pulses have a wide spread in space at $t=0$, requiring a very large and inefficient computational box. To avoid this constraint we implemented the total-field scatteredfield (TFSF) ${ }^{12}$ technique, which allows the incorporation of almost monochromatic sources. The system is divided into two regions: One is the total field (TF) region (where the EM field is the sum of the scattered and the incident field), and the other is the scattered field (SF) region (where only the scattered field is computed). The incident EM wave is launched into the system precisely at the boundaries defined by the TF/SF regions.

The GW is defined as

$$
E_{x}(z, t)=\frac{1}{2}\left(E_{x}^{0} e^{l \frac{\omega_{0}}{c} s} e^{-\left(\frac{s}{\sigma}\right)^{2}}+\text { c.c. }\right),
$$

where $s=z-z_{0}-c t / n_{1}, z_{0}$ being the position where the gaussian field reaches its maximum at $t=0$, and $n_{1}=\sqrt{\varepsilon_{1}}$ corresponds to the refractive index of the illumination region. The parameter $\sigma$ controls both the spectral width and the pulse duration. Without lack of generality $E_{x}^{0}$ is chosen to be a real number. This wave packet carries an instantaneous intensity:

$$
I(z, t)=n_{1} \epsilon_{0} c\left|E_{x}^{0}\right|^{2} \cos \left(\frac{\omega_{0}}{c} s\right)^{2} e^{-2\left(\frac{s}{\sigma}\right)^{2}} .
$$

In the case of linear calculations $\left(\chi^{(3)}=0\right), \sigma$ is chosen so that the wave packet contains all the frequencies of interest. To calculate transmission $\left(T_{L}\right)$ and reflection $\left(R_{L}\right)$ as a function of the wavelength of the incoming light, we project onto diffracted modes in each dielectric half space, so we can distinguish among the different diffracted orders (see Ref. 14 for further details). Additionally, the TFSF method enables the calculation of transmitted and reflected currents within a single simulation: The reflection region is chosen to be the SF region; and the structure along with the transmission

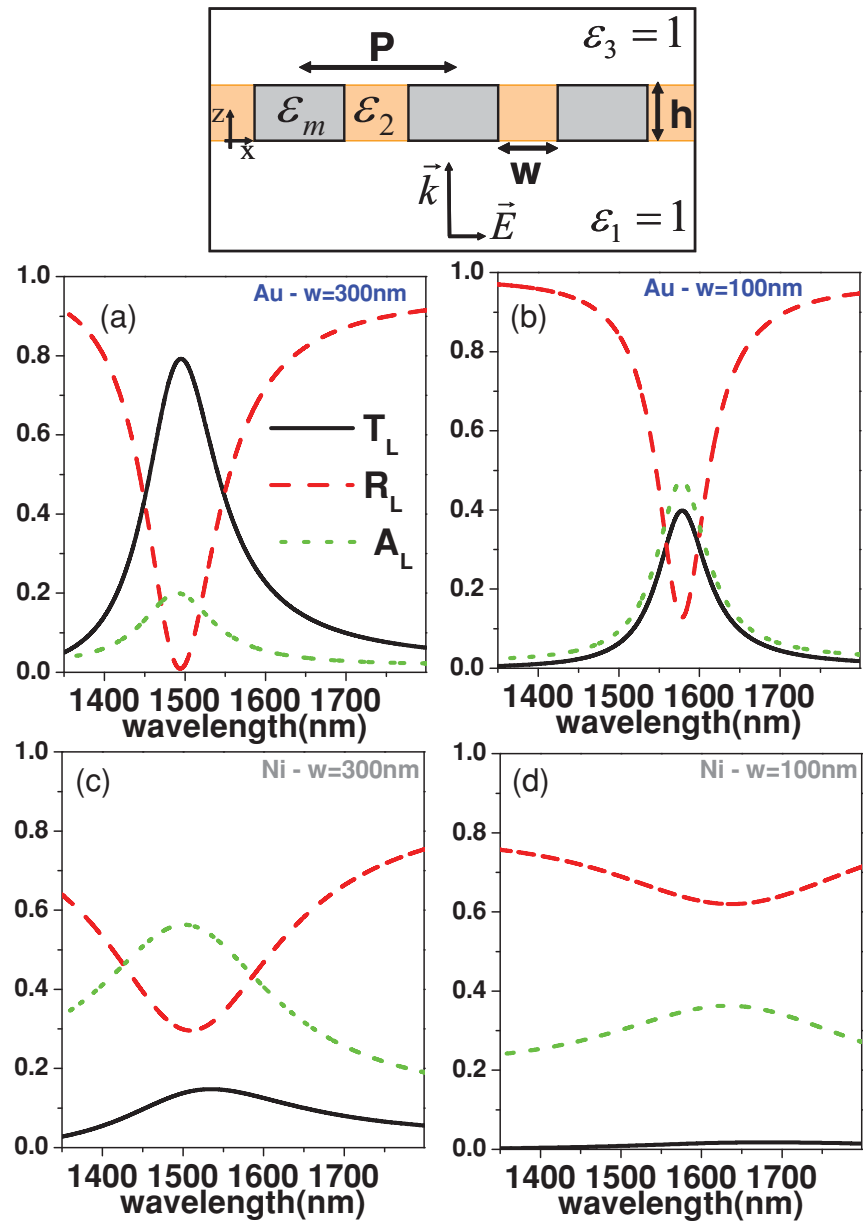

FIG. 1. (Color online) Panels (a) and (b) render transmission, reflection, and absorption through gold metal films pierced by slits periodically arranged, as a function of wavelength. Two different slit widths $w=300 \mathrm{~nm}$ and $w=100 \mathrm{~nm}$ are chosen in panels (a) and (b), respectively. The slits are filled with a dielectric constant $\varepsilon_{2}=10.0$. The rest of geometrical parameters are $P=1200 \mathrm{~nm}$ and $h=185 \mathrm{~nm}$. Panels (c) and (d) correspond to nickel. Top figure: Schematic of the system.

half space cover the TF one. These quantities are calculated integrating the Poynting vector flux through a unit cell, in the frequency domain. In the nonlinear case, transmittance and reflectance, for both the fundamental harmonic $(\mathrm{FH})$ and the third harmonic $(\mathrm{TH})$, are easily obtained by integrating the transmitted and reflected power in a frequency range around the different harmonics, and normalizing to the power of the incident field. The width of the frequency window used in the integration is not important, provided that it is both much smaller than the fundamental frequency and larger than the spectral width of the incident wave packet.

\section{GEOMETRICAL AND MATERIAL PROPERTIES}

The geometrical parameters of the slit arrays (see top panel of Fig. 1) are selected so that a transmission resonance appears at telecom wavelengths $(\sim 1500 \mathrm{~nm})$. The location of this resonance is mostly dictated by the period (which is chosen to be $P=1200 \mathrm{~nm}$ ), and the film thickness ${ }^{15}$ (in this case $h=$ $185 \mathrm{~nm})$. We consider free-standing systems $\left(\varepsilon_{1}=\varepsilon_{3}=1\right)$. 
Since the nonlinear material filling the slits is chosen to be isotropic, homogeneous, and dispersionless in frequency, the EM field responds instantaneously. Materials with high $\varepsilon$ values usually possess also large values of $\chi^{(3)}$, following Miller's rule. ${ }^{1}$ Chalcogenide glasses such as $\mathrm{As}_{2} \mathrm{Se}_{3}$ fit well the later requirements ${ }^{16-20}$ at telecom, and additionally, these glasses feature low linear and two-photon absorption (TPA) at infrared wavelengths, where they are transparent. However, in real experiments these materials support a maximum change in the refractive index $\Delta n \approx \Delta \varepsilon / 2 \sqrt{\varepsilon} \sim 0.0001$ before being damaged. This value is too small for nonlinear applications based on a strong change of the local dielectric constant, as the OKE considered here. As an alternative to chalcogenides, semiconductors such as GaAs appear as good candidates, with an index change threshold four orders of magnitude higher than the one for chalcogenides, ${ }^{21,22}$ while still featuring high $\chi^{(3)}$. In any case, appropriate candidates for real experiments must have both high $\varepsilon$ and $\chi^{(3)}$, and also must behave as Kerrtype materials with low absorption in a wide range of local intensities and wavelengths. For the sake of illustration in what follows we take $\varepsilon=10.0$ and $\chi^{(3)} \approx 3.682 \times 10^{-19}\left(\mathrm{~m}^{2} / \mathrm{V}^{2}\right)$ (from Miller's rule) which are typical semiconductor values.

Concerning metals, both gold and nickel are considered in this paper, because they are representative cases of low- and high-absorbing metals, respectively. The dielectric constant, $\varepsilon_{m}$, is taken from the experimental values tabulated in Refs. 23 and 24, and conveniently fitted into Drude-Lorentz formulas. ${ }^{14,25}$ In this work, we neglect the nonlinear response of metals. Note that the change in the metal dielectric constant due to OKE would be orders of magnitude lower than the dielectric constant of metals at telecom, making a noticeable OKE related effect unlikely. In contrast, if the considered dielectric presents a strong nonlinear coefficient, $\mathrm{TH}$ generation and emission from the metal itself are expected to be hidden by the high-index medium response, according to recent FWM experiments by Renger et al. ${ }^{26}$

\section{LINEAR RESPONSE}

Let us start with the linear optical response $\left(\chi^{(3)}=0\right)$. Figure 1 depicts the zeroth-order transmittance $\left(T_{L}\right)$, reflectance $\left(R_{L}\right)$, and absorbance $\left(A_{L}\right)$, through gold slit arrays (upper panels) and nickel ones (lower panels). Two different slit widths have been considered: $w=300 \mathrm{~nm}$ and $w=100$ $\mathrm{nm}$ (left and right panels, respectively).

For gold systems, panels (a) and (b) in Fig. 1 show resonances emerging at $\lambda \approx 1550 \mathrm{~nm}$, while the simulations for nickel present an almost flat spectra [panels (c) and (d)]. Resonances in gold originate from the hybridization of surface plasmon polaritons, and a localized Fabry-Perot resonance. ${ }^{15,27}$ As we will see next, the nonlinear response is determined by both the intensity and width in frequency of the different spectral features in the linear regime. For slit arrays, transmission features strongly depend on both the width of the aperture and the optical properties of the metal (as in two-dimensional arrays of holes ${ }^{14,28}$ ). In particular, absorption reduces the time photons stay inside the apertures, and this is evidenced in the full width at half maximum of the transmission peaks, $\Delta \lambda$. The fact that peaks in nickel are much wider than those in gold for the same geometrical parameters suggests that absorption losses are dominant in the former case. In the same way, radiation losses are expected to depend mainly on the width of the opening. Hence, when studying $\Delta \lambda$ for slit arrays in gold where actual transmission resonances take place, we find wider transmittance peaks for wider openings because coupling with radiation is easier in them. Additionally, as photons stay less time inside wide apertures, absorbance is also lower in systems with wider openings, as can be seen in panels (a) and (b) in Fig. 1.

\section{NONLINEAR RESPONSE}

Next, we will characterize the nonlinear response of the slit arrays investigated in Fig. 1. We describe the nonlinear behavior for wavelengths fixed at transmittance maxima for gold, where intense EM fields are expected to occur. As slit arrays in nickel do not show resonant behavior, for a given slit width the same wavelengths are chosen for nickel and gold (see inset in each panel of Fig. 2).

\section{A. Optical Kerr effect}

For these nonlinear calculations we illuminate the structure with a $820 \mathrm{fs} \mathrm{GW}(\sigma \approx 84 \mu \mathrm{m})$, so that the spectral width
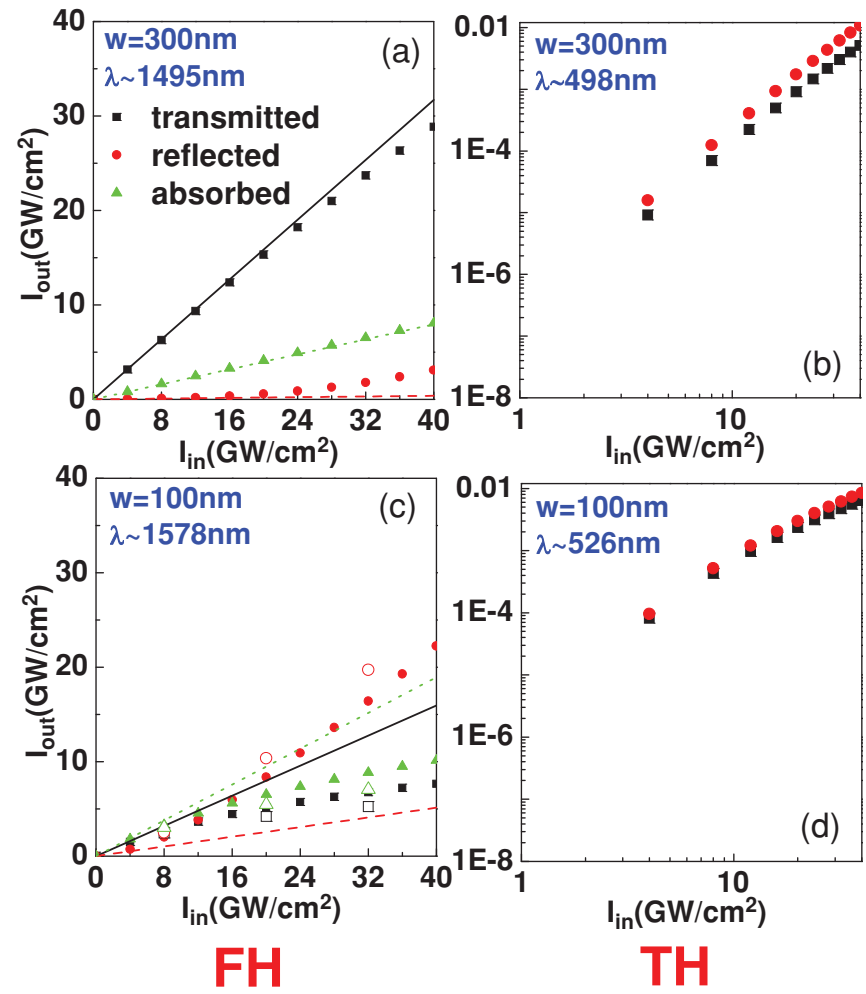

FIG. 2. (Color online) Slit arrays in gold $(P=1200 \mathrm{~nm}$, $h=185 \mathrm{~nm}$ ). Panels (a), (c) depict the calculated FH nonlinear transmitted and reflected intensities, along with the absorption (symbols), as a function of the maximum instantaneous input intensity, at $\lambda \sim 1495 \mathrm{~nm}$ and $\lambda \sim 1578 \mathrm{~nm}$, respectively. Additionally, the corresponding linear curves are depicted with thin lines. Panels (b) and (d) show TH emission (double logarithm scale) at $\lambda \sim 498 \mathrm{~nm}$ and $\lambda \sim 526 \mathrm{~nm}$, respectively. The empty symbols in (c) render the results for a plane wave. 

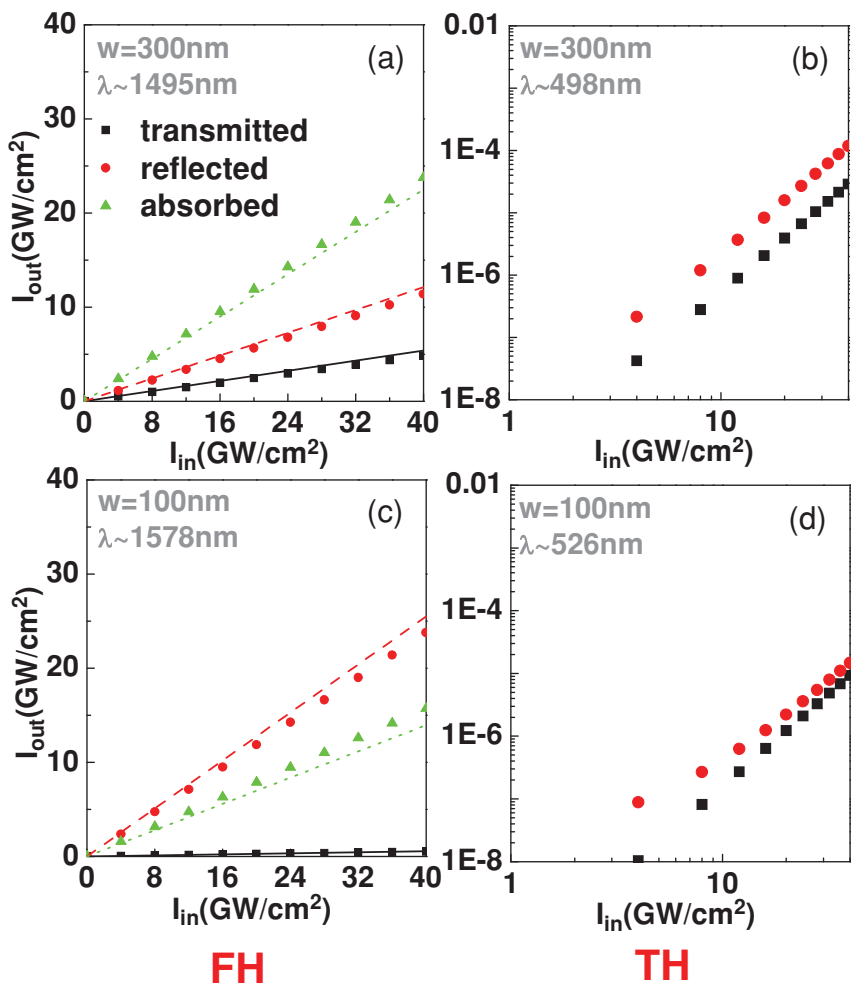

FIG. 3. (Color online) Slit arrays in nickel $(P=1200 \mathrm{~nm}$, $h=185 \mathrm{~nm}$ ). Panels (a), (c) depict the calculated FH nonlinear transmitted and reflected intensities, along with the absorption (symbols), as a function of the the maximum instantaneous input intensity, at $\lambda=1495 \mathrm{~nm}$ and $\lambda=1578 \mathrm{~nm}$, respectively. Additionally, the corresponding linear curves are depicted with thin lines. Panels (b) and (d) show the corresponding TH calculations at $\lambda \sim 498 \mathrm{~nm}$ and $\lambda \sim 526 \mathrm{~nm}$, respectively.

is $\Delta \lambda \approx 10 \mathrm{~nm}$. The left panels of Fig. 2 and Fig. 3 show the results of the FH transmitted and reflected intensities as a function of the maximum instantaneous input intensity $\left(I_{\text {in }}\right)$, for gold and nickel systems, respectively. Two slit widths are considered: (a) $w=300 \mathrm{~nm}$ and (c) $w=100 \mathrm{~nm}$. In the same figures thin lines represent transmitted, reflected, and absorbed intensities obtained from extrapolation of the linear case. In arrays in gold (Fig. 2), when $I_{\text {in }}$ increases the transmitted output intensity decreases to values lower than those expected from the linear transmittance. This reduction is more clear for $w=100 \mathrm{~nm}$. Interestingly, this transmitted output "limitation" occurs mostly at the expense of the reflected current, so the absorption levels are restrained to values $<10 \mathrm{GW} / \mathrm{cm}^{2}$ within the input intensity range here studied. This reduction in transmitted output can be understood as follows: OKE increases the dielectric constant of the Kerr-type dielectric filling the slits [Eq. (2)]. Therefore, the effect of increasing the input intensity can, in a first approximation, be understood by a rigid redshift of the linear spectrum. As we consider wavelengths for maximum transmittance in the linear case, the intensity-induced shift reduces the differential transmittance. It is worth mentioning that by choosing wavelengths that do not correspond to maxima in the linear transmission, a different optical response rather than optical limiting could be obtained.
For instance, optical switching has been theoretically predicted close to transmission minima. ${ }^{29}$

Figures 3(a) and 3(c) depict FH emission for nickel systems with $w=300 \mathrm{~nm}$ and $w=100 \mathrm{~nm}$, respectively. Notice that, for nickel, $T$ and $R$ hardly deviate from their values in the linear regime. As we show later, for the considered intensities the induced change of the dielectric constant inside the slits is much smaller than for gold. Moreover, as the linear transmission and reflection spectra present very smooth variations, the small nonlinear induced change in the dielectric constant translates into an even smaller change in the scattering coefficients.

Phenomena related to OKE occur due to local variations of the refractive index modulated by the average of the electric field intensity [Eq. (2)]. Since this change in the refractive index is not very large, for nonlinear dielectrics in bulk it is common to approximate it as $\Delta n=n_{2} I_{\text {in }}$, where $n_{2}=\frac{3 \chi^{(3)}}{4 n^{2} c \varepsilon_{0}}$ is the Kerr coefficient, with $n=\sqrt{\varepsilon}$ and $c$ the speed of light. However, this approximation assumes that no field enhancement takes place in the structure. If a nonlinear

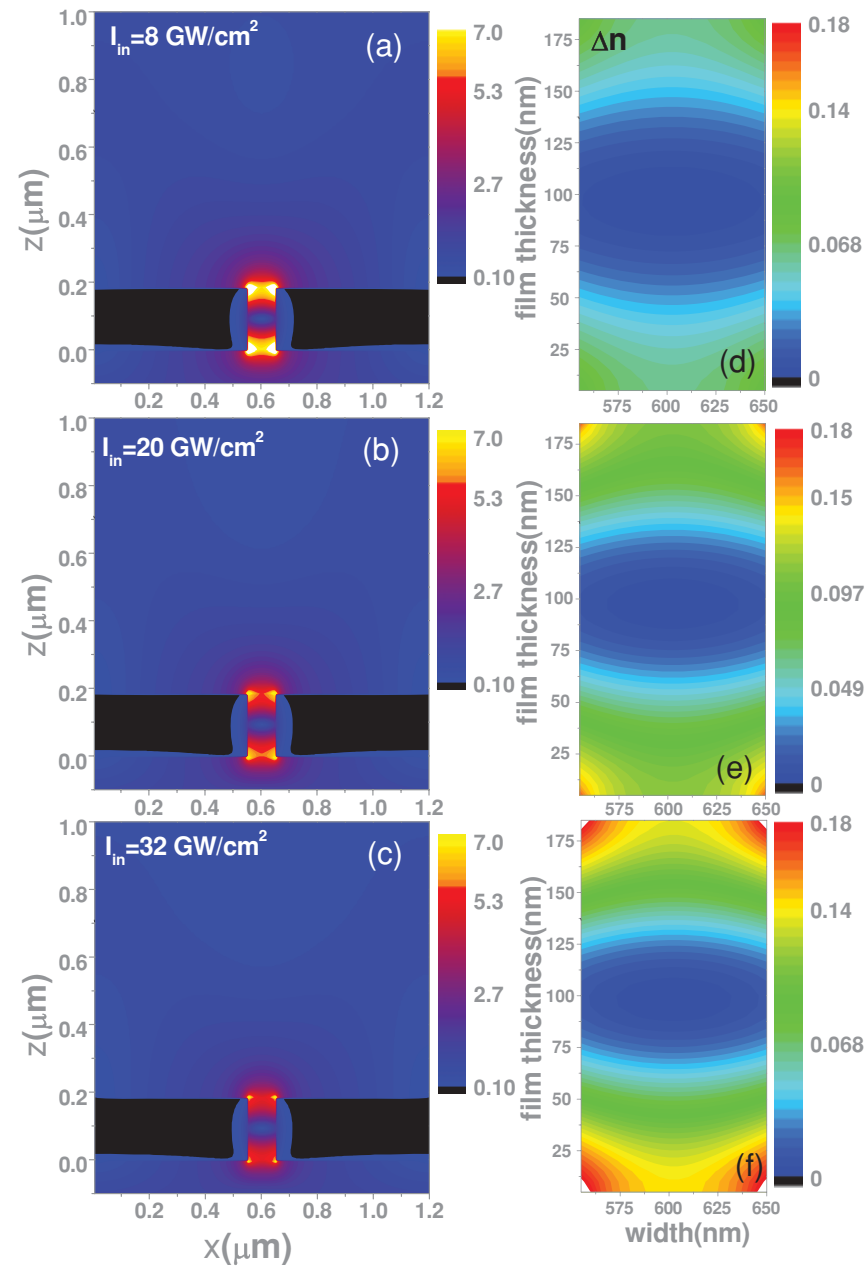

FIG. 4. (Color online) For a slit array in gold $(P=1200 \mathrm{~nm}$, $h=185 \mathrm{~nm}, w=100 \mathrm{~nm})$ illuminated with a PW $(\lambda \sim 1578 \mathrm{~nm})$, panels (a), (b), (c) display $|\vec{E}(x, z)| /\left|\vec{E}_{0}\right|$ for a unit cell, for three different values of $I_{\text {in }}: 8 \mathrm{GW} / \mathrm{cm}^{2}, 20 \mathrm{GW} / \mathrm{cm}^{2}$ and $32 \mathrm{GW} / \mathrm{cm}^{2}$, respectively. The corresponding local change in the refractive index inside the slits, $\Delta n(x, z)$, is depicted in panels (d), (e), and (f). 
dielectric filling a slit array behaved as a dielectric in bulk, then (i) the electric field intensity should be homogeneous inside the slits, and (ii) the field enhancement should be constant and close to unity for all wavelengths.

Let us investigate whether the above postulations are fulfilled in the chosen structures. First, we consider another case where the illumination source is a plane wave $(\mathrm{PW})$. To simulate this PW source $\left(e^{l \omega_{0} s / c}\right)$, an exponentially increasing function is employed to switch on the source from zero to the stationary state at the TF/SF boundaries. This smooth "switching on" is necessary because otherwise there would be EM fields different from zero at $t=0$ along these boundaries, provoking instabilities in the FDTD time evolution. Once the stationary state is reached, this source carries an instantaneous intensity identical to that of a $\mathrm{GW}$ at maximum. For comparison, Fig. 2(c) renders with empty symbols the FH intensity obtained with this source. We get slightly different results, which highlights an important consequence when dealing with nonlinear materials: The nonlinear optical response not only depends on the geometrical parameters, materials, and intensity of the impinging light considered, but also on the spatial profile of the beam and its temporal dependence. These differences may be attributed to "memory effects," precisely related to the particular EM field time evolution.
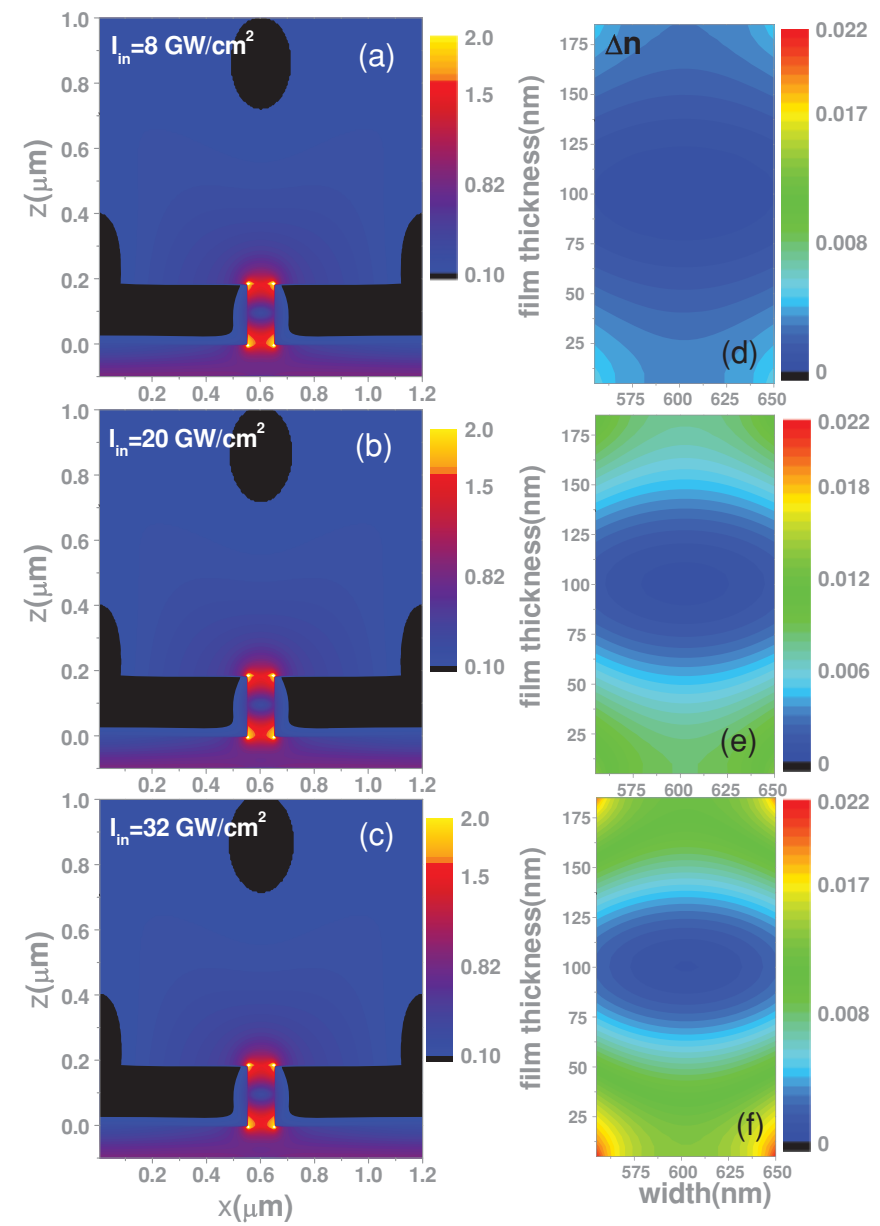

FIG. 5. (Color online) Similar study to the one shown in Fig. 4 but for a slit array in nickel $(P=1200 \mathrm{~nm}, h=185 \mathrm{~nm}, w=100 \mathrm{~nm})$ illuminated with a PW $(\lambda=1578 \mathrm{~nm})$.
Next, Fig. 4 and Fig. 5 show a field enhancement coefficient defined as $\alpha=|\vec{E}(x, z)| /\left|\vec{E}_{0}\right|$ (left panels) and the corresponding local change in the refractive index only inside the slits, $\Delta n(x, z)$ (right panels), for slit arrays in gold and in nickel, respectively. Three different instantaneous input intensities are considered, and the slit width is chosen to be $w=100 \mathrm{~nm}$. The local change of the refractive index inside the slits is computed from the values of the electric field amplitude at the stationary state. These figures show that for gold systems both the field enhancement coefficient and $\Delta n(x, z)$ strongly depend on the incident intensity (where different patterns are obtained for each incident intensity), whereas the panels for nickel systems present negligible changes in the pattern for any $I_{\text {in }}$. Additionally, both metals present inhomogeneous $\alpha(x, z)$ field enhancements with high intensity lobes at the entrance and at the exit of the slits, and a field decrease through the middle. These results indicate that OKE in gold systems arises from two effects: (i) linear transmission spectra depend on the incident wavelength and (ii) $\alpha(x, z)$ are inhomogeneous spatial coefficients that depend on the incident intensity. Therefore, when the incident intensity increases, so does the field enhancement coefficient, and this translates into an increase of $\Delta n(x, z)$. As an increase of the dielectric constant in the linear regime produces a rigid redshift of the linear spectrum, only for gold systems where sharp transmission features take place, the nonlinear response deviates rapidly from the linear regime. On the contrary, in nickel systems, neither do the linear spectra depend on the wavelength, nor does the field enhancement coefficient depend on the incident intensity, and as a result, the nonlinear optical response associated with the OKE hardly deviates from the linear regime.
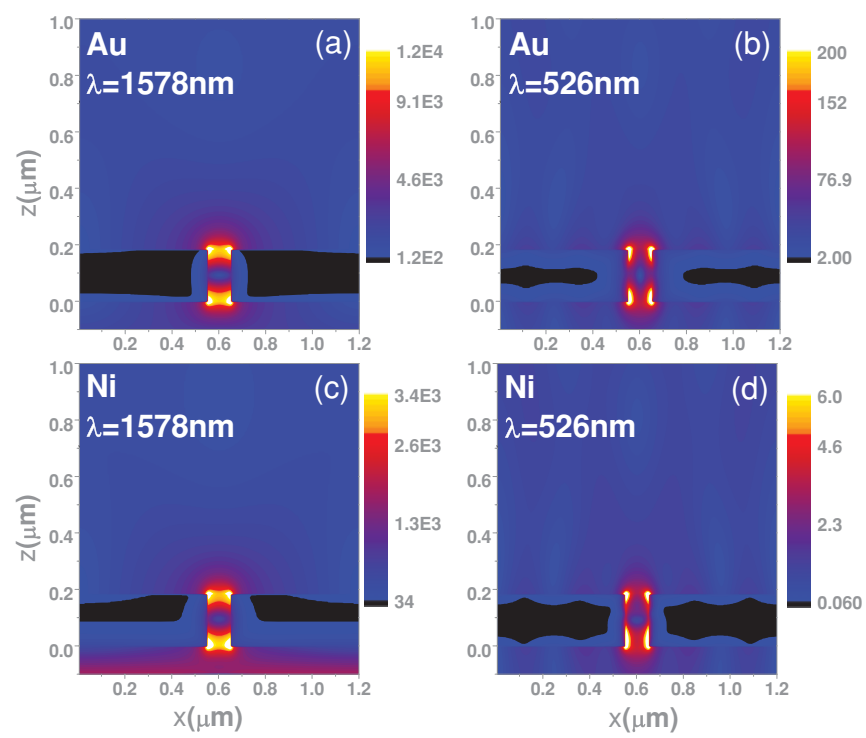

FIG. 6. (Color online) Electric field amplitude in a slit array at $\lambda=1578 \mathrm{~nm}(\mathrm{FH})$ and $\lambda=526 \mathrm{~nm}(\mathrm{TH})$. Panels (a) and (b) are for gold and (c) and (d) for nickel. The incident intensity is $I_{\text {in }}=32 \mathrm{GW} / \mathrm{cm}^{2}, w=100 \mathrm{~nm}$, and the rest of geometrical parameters as in Fig. 1. 


\section{B. Third-harmonic generation}

In this section we investigate $\mathrm{TH}$ emission arising from the nonlinear dielectric filling the slits. As in Sec. V A, Fig. 2 corresponds to calculations for slit arrays in gold, and Fig. 3 to nickel systems. Panels (b) and (d) correspond to TH calculations. For gold systems we observe that TH currents are several orders of magnitude smaller than either the input intensity or the corresponding FH values [depicted with full symbols in panels (a) and (c)]. For nickel systems, despite FH transmission not deviating from what would be expected if the system responded linearly [panels (a) and (c)], the appearance of $\mathrm{TH}$ radiation unveils third-order nonlinear processes taking place inside the slits. Interestingly, despite $R_{L}$ in the linear regime at the corresponding wavelength where $\mathrm{TH}$ emission takes place, $\lambda=526 \mathrm{~nm}$, being orders of magnitude larger than $T_{L}$, nonlinear calculations in Fig. 2 and Fig. 3 show almost identical $\mathrm{TH}$ radiated currents. For instance, in the linear regime for slits in gold and $w=100 \mathrm{~nm}, R_{L}=0.67$ and $T \approx 2.4 \times 10^{-4}$ at $\lambda=526 \mathrm{~nm}$, while in the nonlinear regime emission into the reflection region is only 1.3 times larger than that into the transmission half space. Because $\mathrm{TH}$ emission goes as the cubed local intensity, it is expected to be a function of both time and space. Figure 6 shows the electric field amplitudes for the $w=100 \mathrm{~nm}$ slits at $I_{\text {in }}=32 \mathrm{GW} / \mathrm{cm}^{2}$. Panels (a) and (b) depict near field in the case of gold at $\lambda=1578 \mathrm{~nm}(\mathrm{FH})$ and $\lambda=526 \mathrm{~nm}(\mathrm{TH})$, respectively. The bottom panels, (c) and (d), show the same but for nickel. This figure suggests that if the structure is designed so that $\mathrm{FH}$ is efficiently transmitted, the field at the exit of slits must be comparable to the fields at the entrances. In that case, transmission coefficients at the wavelength where $\mathrm{TH}$ generates do not play an important role because light is generated directly close to the slit exits. Besides, although gold and nickel show similar distributions of the electric field amplitudes, local intensity is much higher in gold slits than in nickel ones [compare panels (a) and (c)].

\section{CONCLUSIONS}

In conclusion, we have theoretically analyzed the nonlinear optical response of arrays of slits, drilled on either gold or nickel films, filled with a third-order nonlinear material. $A$ priori, all of them should display optical effects related to $\mathrm{OKE}$ and TH generation. However, these effects strongly depend on the material properties of both the dielectric filling the slits and the metal chosen. We attribute to OKE the behavior found in the transmitted and reflected currents calculated for the slit arrays in gold. However, in nickel OKE is not strong enough for boosting noticeable modifications in the optical spectra. This shows that the efficiency of the nonlinear response strongly depends on the intrinsic losses, and that losses due to absorption play the most important role in these systems. Furthermore, TH generation is observed for both metals. The emission of photons at the third harmonic of near-infrared and telecom wavelengths opens the door for ultraviolet and optical light sources from nanoscale areas.

\section{ACKNOWLEDGMENTS}

We acknowledge support from the Spanish Ministry of Science and Innovation under Projects No. MAT2008-06609C02 and No. CSD2007-046-Nanolight.es. The authors acknowledge L. Novotny, F. de León-Pérez, and J. A. Porto for fruitful discussions.
${ }^{1}$ R. W. Boyd, Nonlinear Optics (Academic Press, San Diego, 2003). ${ }^{2}$ G. A. Wurtz, R. Pollard, and A. V. Zayats, Phys. Rev. Lett. 97, 057402 (2006).

${ }^{3}$ G. A. Wurtz and A. V. Zayats, Laser and Photonics Rev. 2, 125 (2008).

${ }^{4}$ J. A. Porto, L. Martín-Moreno, and F. J. García-Vidal, Phys. Rev. B 70, 081402 (2004).

${ }^{5}$ S. Xie, H. Li, H. Xu, X. Zhou, S. Fu, and J. Wu, Opt. Commun. 283, 998 (2010).

${ }^{6}$ S. Xie, H. Li, H. Xu, X. Zhou, S. Fu, and J. Wu, Sci. China: Phys. Mech. Astron. 53, 474 (2010).

${ }^{7}$ C. Min, P. Wang, X. Jiao, Y. Deng, and H. Ming, Opt. Express 15, 9541 (2007).

${ }^{8}$ M. A. Vincenti, A. D’Orazio, M. Buncick, N. Akozbek, M. J. Bloemer, and M. Scalora, J. Opt. Soc. Am. B 26, 301 (2009).

${ }^{9}$ C. Min, P. Wang, X. Jiao, Y. Deng, and H. Ming, Opt. Express 15, 12368 (2007).

${ }^{10}$ C. Min, P. Wang, C. Chen, Y. Deng, Y. Lu, H. Ming, T. Ning, Y. Zhou, and G. Yang, Opt. Lett. 33, 869 (2008).

${ }^{11}$ M. A. Vincenti, D. de Ceglia, V. Roppo, and M. Scalora, Opt. Express 19, 2064 (2011).
${ }^{12}$ A. Taflove and S. Hagness, Computational Electrodynamics: The Finite-Difference Time-Domain Method (Artech House, Boston, 2000).

${ }^{13}$ P. Tran, Phys. Rev. B 52, 10673 (1995).

${ }^{14}$ S. G. Rodrigo, F. J. García-Vidal, and L. Martín-Moreno, Phys. Rev. B 77, 075401 (2008).

${ }^{15}$ F. J. García-Vidal and L. Martín-Moreno, Phys. Rev. B 66, 155412 (2002).

${ }^{16}$ N. Bloembergen, W. Burns, and M. Matsuoka, Opt. Commun. 1, 195 (1969).

${ }^{17}$ V. Ta'eed, N. J. Baker, L. Fu, K. Finsterbusch, M. R. E. Lamont, D. J. Moss, H. C. Nguyen, B. J. Eggleton, D.-Y. Choi, S. Madden et al., Opt. Express 15, 9205 (2007).

${ }^{18}$ G. P. Agrawal, Non-Linear Fiber Optics (Academic Press, San Diego, 2001).

${ }^{19}$ J. Troles, F. Smektala, M. Guignard, P. Houizot, V. Nazabal, H. Zeghlache, G. Boudebs, and V. Couderc, in Transparent Optical Networks, 2005, Proceedings of 20057 th International Conference, Vol. 2 (IEEE, Barcelona, Spain, 2005), pp. 242-244.

${ }^{20}$ J. Troles, F. Smektala, G. Boudebs, A. Monteil, B. Bureau, and J. Lucas, Opt. Mater. 25, 231 (2004). 
${ }^{21}$ W. C. Hurlbut, Y.-S. Lee, K. L. Vodopyanov, P. S. Kuo, and M. M. Fejer, Opt. Lett. 32, 668 (2007).

${ }^{22}$ L. Brzozowski, E. H. Sargent, A. S. Thorpe, and M. Extavour, Appl. Phys. Lett. 82, 4429 (2003).

${ }^{23}$ E. D. Palik, Handbook of Optical Constants of Solids (Academic Press, New York, 1985).

${ }^{24}$ E. D. Palik, Handbook of Optical Constants of Solids II (Academic Press, Boston, 1991).

${ }^{25}$ F. Hao and P. Nordlander, Chem. Phys. Lett. 446, 115 (2007).
${ }^{26}$ J. Renger, R. Quidant, and L. Novotny, Opt. Express 19, 138720 (2011).

${ }^{27}$ F. J. Garcia-Vidal, L. Martin-Moreno, T. W. Ebbesen, and L. Kuipers, Rev. Mod. Phys. 82, 729 (2010).

${ }^{28}$ F. Przybilla, A. Degiron, J.-Y. Laluet, C. Genet, and T. W. Ebbesen, J. Opt. A 8, 458 (2006).

${ }^{29}$ S. Carretero-Palacios, A. Minovich, D. N. Neshev, Y. S. Kivshar, F. J. Garcia-Vidal, L. Martin-Moreno, and S. G. Rodrigo, Opt. Lett. 35, 4211 (2010). 